\title{
Multiplexed Detection Strategy for Bladder Cancer MicroRNAs Based on Photonic Crystal Barcodes
}

Xiaowei Wei ${ }^{1}$, Feika Bian ${ }^{2}$, Xiaoxiao Cai ${ }^{1}$, Yu Wang ${ }^{2}$, Lijun Cai ${ }^{2}$, Jian Yang ${ }^{3}$, Yefei $\mathrm{Zhu}^{1, *}$, Yuanjin Zhao ${ }^{2, *}$

${ }^{1}$ Laboratory Medicine Center, Second Affiliated Hospital of Nanjing Medical University, Nanjing, 210011, China.

2 State Key Laboratory of Bioelectronics, School of Biological Science and Medical Engineering, Southeast University, Nanjing, 210096, China.

${ }^{3}$ Department of Urology, Second Affiliated Hospital of Nanjing Medical University, Nanjing, 210011, China.

E-mail: zhuyf@njmu.edu.cn, yjzhao@seu.edu.cn 

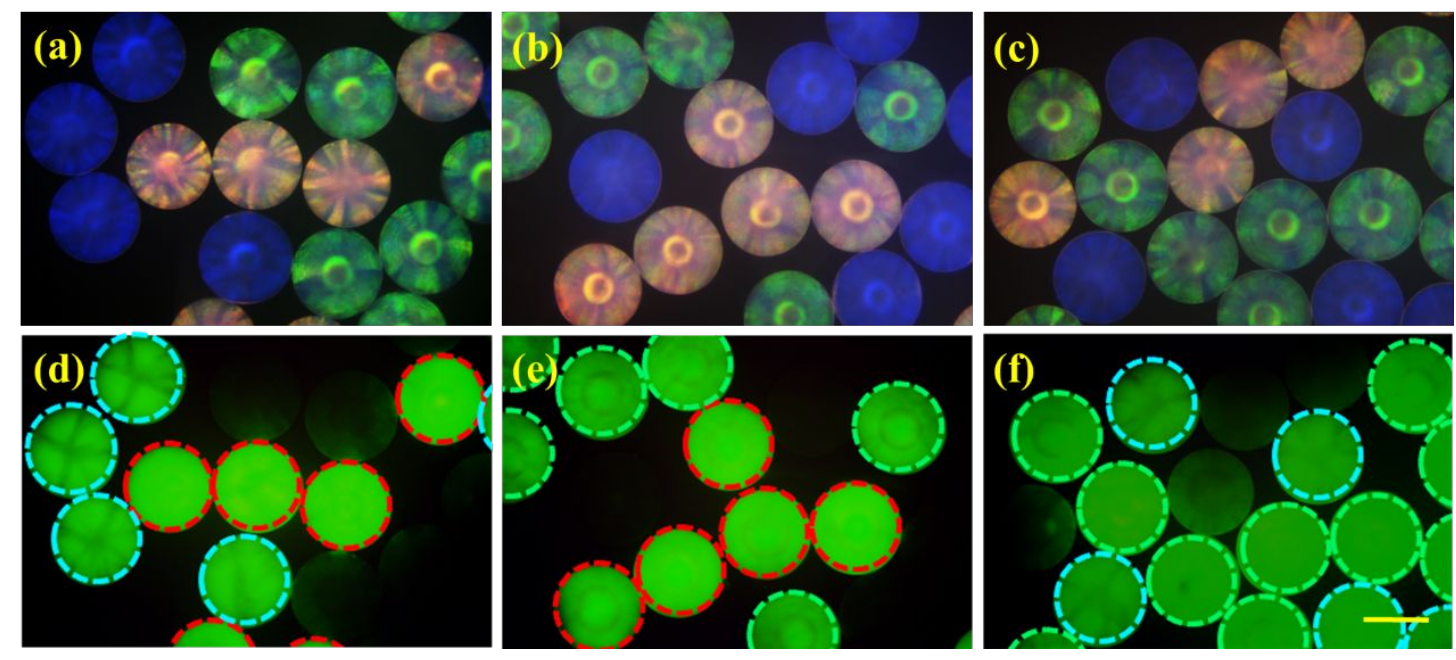

Figure. S1. Images of optical microscope (a-c) and fluorescence (d-f) after three kinds of target miRNAs captured by PhC barcodes; Scale is $200 \mu \mathrm{m}$.

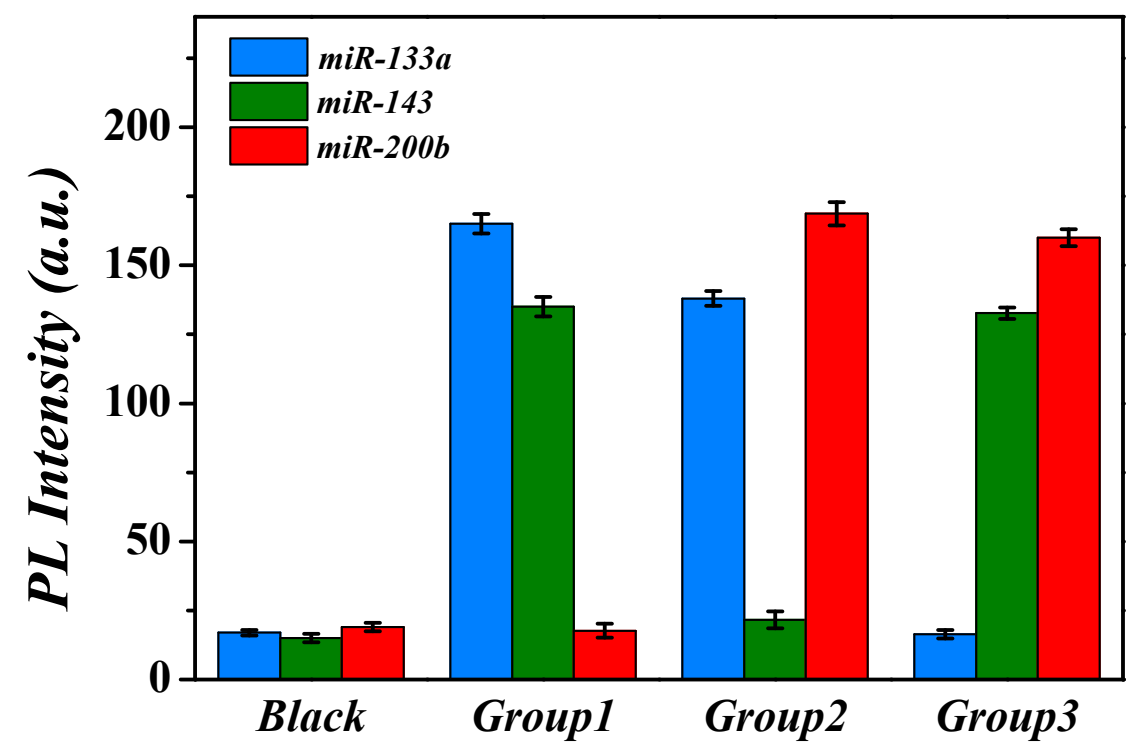

Figure. S2. Fluorescence intensity analysis of $\mathrm{PhC}$ barcodes incubated with three kinds of target miRNAs Error bars represent standard deviations.
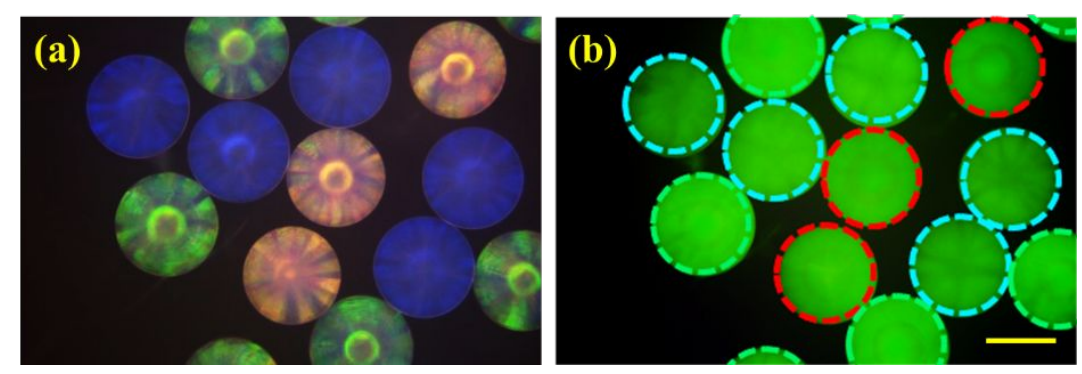

Figure. S3. Images of optical microscope (a) and fluorescence (b) after three kinds of target miRNAs captured by PhC barcodes; Scale is $200 \mu \mathrm{m}$. 


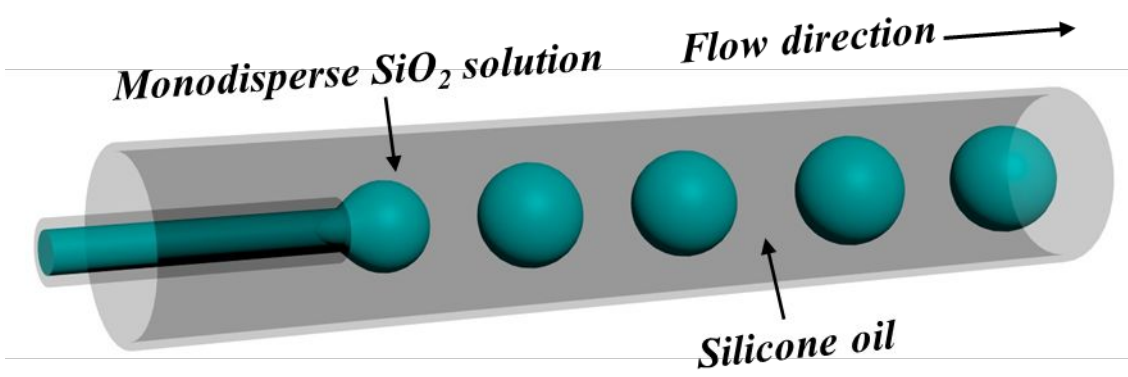

Figure. S4. Schematic diagram of the droplet template prepared by microfluidic technique.

Table S1. Sequences for the probes, targets, initiators and hairpins.

\begin{tabular}{|c|c|c|}
\hline \multicolumn{2}{|c|}{ Oligonucleotides } & Sequence $\left(5^{\prime}-3^{\prime}\right]$ \\
\hline \multirow{6}{*}{$\operatorname{miR}-133 a$} & probe1 & Amino C6-TTTTTTCAGCTGGTTGAAGG \\
\hline & target 1 & UUUGGUCCCCUUCAACCAGCUG \\
\hline & $1 \mathrm{bm}$ & UUUGGUCCCCUCCAACCAGCUG \\
\hline & $3 \mathrm{bm}$ & UUUAGUCCCCUCCAACCAACUG \\
\hline & $5 \mathrm{bm}$ & UUUAGUCUCCUCCAAUCAACUG \\
\hline & initiator1 & GGACCAAA AACCCTAACCCT \\
\hline \multirow{6}{*}{$\operatorname{miR}-143$} & probe2 & Amino C6-TTTTTTGAGCTACAGTGCT \\
\hline & target2 & UGAGAUGAAGCACUGUAGCUC \\
\hline & $1 \mathrm{bm}$ & UGAGAUGAAGUACUGUAGCUC \\
\hline & $3 \mathrm{bm}$ & UGGGAUGAAGUACUGUAGUUC \\
\hline & $5 \mathrm{bm}$ & UGGGAUAAAGUACUAUAGUUC \\
\hline & initiator2 & TCATCTCA AACCCTAACCCT \\
\hline \multirow{6}{*}{$\mathrm{miR}-200 \mathrm{~b}$} & probe3 & Amino C6-TTTTTTTCATCATTACCAGGC \\
\hline & target3 & UAAUACUGCCUGGUAAUGAUGA \\
\hline & $1 \mathrm{bm}$ & UAAUACUGCCUAGUAAUGAUGA \\
\hline & $3 \mathrm{bm}$ & UAACACUGCCUAGUAAUGGUGA \\
\hline & $5 \mathrm{bm}$ & UAACACUACCUAGUAGUGGUGA \\
\hline & initiator3 & AGTATTA AACCCTAACCCT \\
\hline \multicolumn{2}{|c|}{$\mathrm{H} 1$} & FAM-ACTTTGAACCCTAGGGTTAGGGTT \\
\hline \multicolumn{2}{|c|}{$\mathrm{H} 2$} & AGGGTTCAAAGTAACCCTAACCCT-FAM \\
\hline
\end{tabular}

